USTC-ICTS-07-23

\title{
Intersecting non-SUSY branes and closed string tachyon condensation
}

\author{
J. X. Lud1, Shibaji Roy 2 , Zhao-Long Wangd 3 and Rong-Jun Wuat \\ a Interdisciplinary Center for Theoretical Study \\ University of Science and Technology of China, Hefei, Anhui 230026, China \\ ${ }^{b}$ Saha Institute of Nuclear Physics, 1/AF Bidhannagar, Calcutta-700 064, India
}

\begin{abstract}
Following [1] we here consider the supergravity solutions representing the charged non-supersymmetric $p$-brane (for $1 \leq p \leq 6$ ) intersecting with chargeless nonsupersymmetric 1-brane and 0-brane of type II string theories. We show how these solutions nicely interpolate between black p-branes and the Kaluza-Klein "bubble of nothing" (BON) by continuously varying some parameters characterizing the solutions from one set of values to another. By performing a time symmetric general bubble initial data analysis, we show that the interpolation implies a possible transition from black $p$-branes to KK BON only for $p \leq 4$, as in these cases there exist locally stable static bubbles under certain conditions. However, for $p>4$, black branes always decay into dynamical bubbles. Contrary to what is known in the literature, we argue that under certain conditions, the mechanism causing this transition to stable static bubbles can be the closed string tachyon condensation for all $p \leq 4$. We also show that the configurations indeed contain (chargeless) F-strings required for the closed string tachyons to appear causing the transitions to occur.
\end{abstract}

\footnotetext{
${ }^{1}$ E-mail: jxlu@ustc.edu.cn

${ }^{2}$ E-mail: shibaji.roy@saha.ac.in

${ }^{3}$ E-mail: zlwang4@mail.ustc.edu.cn

${ }^{4}$ E-mail: rjwu@mail.ustc.edu.cn
} 


\section{Introduction}

In the eighties, while discussing the gravitational properties of certain soliton solutions in five dimensional Kaluza-Klein (KK) gravity, Gross and Perry [2] observed that there exists a two-parameter family of spherically symmetric solutions in this theory. This solution interpolates between the Schwarzschild metric and a soliton solution. The Schwarzschild metric has a singularity masked by a regular horizon while the soliton is non-singular. In between, the configuration has a naked singularity. They interpreted the configuration as describing the exterior geometry of a collapsing star in parameter space, therefore avoiding the issue of naked singularity appearing in the configuration. But they were puzzled by the non-uniqueness of the exterior geometry of a massive object in the KK theory. As a result, the final state of collapsing matter appears to have two possibilities: a collapsing star could end up in a black hole described by the Schwarzschild metric, or it might end up as a soliton, a totally non-singular metric. The soliton as we know now is a variant of Witten's "bubble of nothing" (BON) [3], namely, the static bubble.

A similar family of solutions as we will show also exist in supergravities, the low energy string theories. These are the specific class of intersecting non-susy branes of type II string theories, namely, the charged non-supersymmetric $p$-brane (for $1 \leq p \leq 6$ ) intersecting with chargeless non-supersymmetric 1-brane and 0-brane of type II string theories. These solutions also nicely interpolate between black $p$-branes and the non-singular static KK BON by continuously varying some parameters characterizing the solutions from one set of values to another. Again in between, the configuration has a naked singularity. In this paper we will try to understand the physical meaning of this interpolation.

Recently in a separate development Horowitz [4] has argued that under certain conditions black strings and black branes of type II string theories have dramatic new endpoint: 5 to Hawking evaporation in the form of KK BON [3]. He arrived at this conclusion by applying the closed string tachyonic instability to black strings and branes. Closed string tachyons are known to develop when the fermions in the theory are taken to satisfy antiperiodic boundary conditions along one 6 of the compact directions and the size of the circle becomes of the order of string scale [7, 8]. Adams et. al. [9] have argued that when

\footnotetext{
${ }^{5}$ Generalizing this to charged and uncharged black holes (i.e., $p=0$ case) along with other interesting discussions and potential realistic applications has also been considered recently in [5]. We thank the anonymous referee for bringing this reference to our attention.

${ }^{6} \mathrm{~A}$ circle with antiperiodic fermions has a one-loop Casimir energy which will cause it to contract. We will not address this issue here but refer to its discussion in [4] as well as in [6]. For concreteness, we focus in this paper on the possibility that the circle reaches string scale on or outside the horizon as in [4.
} 
these winding string tachyons are localized they can trigger a topology changing transformation as a consequence of the closed string tachyon condensation. The transitions from black string (branes) to the KK BON, very similar to the interpolation we mentioned, is an application of this process.

In ref. 4. Horowitz started from the standard black fundamental string solution [10, 11 and compactified the string direction. Since the metric in this case is multiplied appropriately with a harmonic function, the size of the string wound along the compact direction varies monotonically from $L$ (where $L$ is the periodicity of the compact direction) to zero as we move along the radial direction from infinity to the singular point. So, at some point in between the size of the circle becomes of the order of string scale and if this occurs on the horizon, then the closed string tachyon condensation causes the circle to pinch off and the resulting state is a bubble which in this case cannot be static but should expand out. Static bubble appeared while considering a similar transition from black F-string containing both F and NS5 charge. However, in order to show that bubble is the end state of this transition, a time symmetric bubble initial data analysis has been performed (in the context of two-charge F-string in space-time dimensions $D=6$ and also with the vanishing dilaton) and it was found that indeed under certain conditions $\left(Q / L^{2} \ll 1\right.$, where $Q$ is the flux associated with the bubble) the bubble can be stable and so, the black F-string in those cases can make a transition to classically stable static bubbles. A similar transition occurs for black $p$-branes as well and was briefly mentioned in [4]. However, it was observed that only for $p=3$ cast 7 the final bubble could be stable and static. We will discuss these cases in more detail in this paper and make new observations regarding the transition from black $p$-branes to stable static bubbles for all $p \leq 4$.

In this paper we first construct the interpolating solutions. For this purpose we start from the supergravity solutions representing the charged non-susy $p$-brane intersecting with chargeless non-susy 1-brane and 0-brane of type II string theories 8 . These solutions will be characterized by four (for $p=1$ ) or five (for $p>1$ ) independent parameters. As we have shown in [1], here also it can be checked that when two (for $p=1$ ) or three (for $p>1$ ) of the four or five parameters characterizing the solutions take some special values, then these intersecting solutions are identical to the black $p$-brane solutions. We also observed [1] that the parameters characterizing the black brane and the related dynamics

\footnotetext{
${ }^{7}$ The other possible cases are M2 and M5 systems or involve more complicated configurations such as the D1-D5 system [6].

${ }^{8}$ Note that for $p=1$ supergravity solution represents charged non-susy 1-brane intersecting with chargeless 0-brane.
} 
are in a different branch of the parameter space from that describing the brane-antibrane annihilation process or the open string tachyon condensation [12, 13]. Although it would be interesting to understand the dynamics of the closed string tachyon condensation in this setup, we, however, point out that the general intersecting solutions (charged nonsusy $p$ brane with chargeless non-susy 1-brane and 0-brane) in fact interpolate between the black $p$-branes and the KK BON through a series of classical solutions. To be specific we find that when two (for $p=1$ ) or three (for $p>1$ ) out of the four (for $p=1$ ) or five (for $p>1$ ) independent parameters characterizing the solutions are continuously varied from one set of values to another the solutions change from the black $p$-branes to the KK BON. There is no need to take a so-called double Wick rotation to obtain the KK bubble and it just corresponds to another point in the parameter space.

Now in order to understand whether the interpolation means a possible transition from black $p$-branes to static KK BON, we go one step further to show that the bubble is locally stable so that it does not evolve further perturbatively. We do this by performing a time symmetric general bubble initial data analysis (Note that we have now non-vanishing dilaton in $D=10$.). Our analysis indicates that only for $p \leq 4$, the initial data can lead to locally stable static bubble under certain conditions (otherwise they lead to dynamical bubbles). So, only for these cases the interpolation has the possibility to be interpreted as the transition from black branes to static KK BON. When $p>4$, the initial data leads either to locally unstable static bubbles or to dynamical bubbles. Therefore, the resulting bubbles are in general dynamical. Bubbles smaller than the locally unstable static bubbles contract while larger ones expand. Since the black $p$-branes and the corresponding bubbles can also be related to each other by the so-called double Wick rotation, their perturbative stability is correlated as discussed in [4]. This provides an independent check of the results obtained through the initial data analysis and we find precise agreement.

Thus it is expected that for $p \leq 4$, there may exist some perturbative or nonperturbative mechanism which leads to the transition from black $p$-branes to static KK BON. This in turn can be understood as the reason for the existence of the interpolating solutions we constructed. As these are the topology changing transitions like what happens for the closed string tachyon condensation we mentioned, one would expect the transition could be triggered by the closed string tachyon condensation 9 discussed in [4]. We find that for $p \leq 4$, there exists a stable static bubble when the ratio $Q / L^{7-p}$ is less than certain maximum value $C_{\max }$ and the black $p$-branes can make a transition to static KK BON by closed string tachyon condensation. When $Q / L^{7-p}>C_{\max }$, the $p$-branes will

\footnotetext{
${ }^{9}$ The correlation between the instabilities of black $p$-branes and the corresponding bubbles seems to support this as well, as we will see.
} 
always decay into dynamical bubbles. However, only for $p=3$, the black D3-brane can decay directly into stable static bubble through closed string tachyon condensation. For $p<3$, the black $p$-branes first decay into unstable static bubble and then under slight perturbation in the right direction can eventually settle in the stable static bubble. But for $p=4$, the black D4-branes first decay into dynamical bubble and then eventually settle in the stable static bubble. So, in all these cases, the interpolation can be interpreted to be caused by the closed string tachyon condensation. For this to hold true, we actually need all the spatial brane directions to be compact and their respective asymptotical sizes to be on the same order of magnitude. For $p=5,6$, as there are no stable static bubbles, the interpolation does not imply a possible transition in parameter space. In these cases the black branes can only decay to dynamical bubbles through closed string tachyon condensation.

We would like to emphasize that if closed string tachyon condensation could be the possible reason for the transition to occur, either to stable static or dynamical bubble, there must be fundamental strings present in the supergravity solutions which is not obvious in our intersecting solutions. In the present context, the net charge of fundamental strings must be zero since the resulting bubble doesn't carry any fundamental string charge coupled to the NSNS 2-form potential and the charge is conserved during the transition. This gives us hint where the fundamental strings can be found. We show that the chargeless non-susy 1-branes present in the intersecting solutions indeed represent the chargeless non-susy F-strings, i.e., the equal number of fundamental strings and anti fundamental strings with zero net charge present in the configuration 10 , which when wound along the compact direction give rise to closed string tachyon causing the transitions to occur. We remark that the singular region pinching off from the regular part of spacetime through closed string tachyon condensation can be viewed as the decoupling in dynamics. This is supported by the evidence found in [14].

This paper is organized as follows. In section 2, we present the construction of the supergravity solutions interpolating between black $\mathrm{D} p$-brane and the KK BON. We give the time symmetric bubble initial data analysis in section 3 . Then we physically interpret the interpolation in terms of the closed string tachyon condensation in section 4. Section 5 discusses the origin of F-strings in the intersecting solutions obtained in section 1 which is necessary for the closed string tachyon condensation to occur. Then we present our conclusion in section 6 .

\footnotetext{
${ }^{10}$ We thank the anonymous referee for suggesting us to clarify this.
} 


\section{Interpolating solutions}

In this section we construct the supergravity solutions interpolating between black $\mathrm{D} p$ brane to static KK BON. As mentioned in the introduction these solutions would be a class of intersecting solutions in supergravity, namely, the charged non-susy $p$-brane intersecting with chargeless non-susy 1-brane and 0-brane. To obtain them we start from the non-isotropic (in $(p-q)$-directions) charged non-susy $p$-brane solutions given in [1]. This solution actually represents charged non-susy $p$-branes intersecting with chargeless $(p-1)$-brane, $(p-2)$-brane, $\ldots$, upto $q$-brane. These solutions were constructed by first delocalizing a non-susy $q$-brane in transverse $(p-q)$ directions and then applying T-duality along the delocalized directions successively [15. But for our purpose here we need the corresponding solutions with $q=0$ and so we write the eq.(7) of [1] with $q=0$ as follows,

$$
\begin{aligned}
d s^{2}= & F^{\frac{p+1}{8}}(H \tilde{H})^{\frac{2}{7-p}}\left(\frac{H}{\tilde{H}}\right)^{\frac{p \delta_{1}}{8}+\frac{(3-p) \sum_{i=1}^{p} \delta_{i+1}}{2(7-p)}}\left(d r^{2}+r^{2} d \Omega_{8-p}^{2}\right) \\
& +F^{-\frac{7-p}{8}}\left(\frac{H}{\tilde{H}}\right)^{\frac{p \delta_{1}}{8}+\sum_{i=1}^{p} \frac{\delta_{i+1}}{2}}\left(-d t^{2}\right) \\
& +F^{-\frac{7-p}{8}}\left(\frac{H}{\tilde{H}}\right)^{\frac{(p-8) \delta_{1}}{8}+\sum_{j=1}^{p} \frac{\delta_{j+1}}{2}} \sum_{i=1}^{p}\left(\frac{H}{\tilde{H}}\right)^{-2 \delta_{i+1}}\left(d x^{i}\right)^{2} \\
e^{2 \phi}= & F^{\frac{3-p}{2}}\left(\frac{H}{\tilde{H}}\right)^{\frac{\delta_{1}}{2}(4-p)-\sum_{i=1}^{p} 2 \delta_{i+1}} \\
F_{[8-p]}= & \hat{Q} \operatorname{Vol}\left(\Omega_{8-p}\right)
\end{aligned}
$$

Here the metric is given in the Einstein frame. The various functions appearing in the solution are defined below,

$$
\begin{aligned}
& F=\left(\frac{H}{\tilde{H}}\right)^{\alpha} \cosh ^{2} \theta-\left(\frac{\tilde{H}}{H}\right)^{\beta} \sinh ^{2} \theta \\
& H=1+\frac{\omega^{7-p}}{r^{7-p}}, \quad \tilde{H}=1-\frac{\omega^{7-p}}{r^{7-p}}
\end{aligned}
$$

where $H$ and $\tilde{H}$ are two harmonic functions and $\alpha, \beta, \theta, \delta_{1}, \delta_{2}, \ldots, \delta_{p+1}$ and $\omega$ are $(p+5)$ integration constants and $\hat{Q}$ is the charge parameter. The parameters here are not all 
independent, but they satisfy the following three relations among themselves [1],

$$
\begin{aligned}
& \alpha-\beta=a \delta_{1} \\
& \frac{1}{2} \delta_{1}^{2}+\frac{1}{2} \alpha\left(\alpha-a \delta_{1}\right)+\frac{2 \sum_{i>j=2}^{p+1} \delta_{i} \delta_{j}}{7-p}=\left(1-\sum_{i=2}^{p+1} \delta_{i}^{2}\right) \frac{8-p}{7-p} \\
& \hat{Q}=(7-p) \omega^{7-p}(\alpha+\beta) \sinh 2 \theta
\end{aligned}
$$

where $a=-3 / 2$. Using the above relations we can eliminate three of the $(p+6)$ parameters mentioned and therefore, the solution is characterized by $(p+3)$ independent parameters. From the isometry of the solutions given in (1), it is clear that they actually represent charged (since $F_{[8-p]}$ is non-zero) non-susy $p$-brane intersecting with chargeless (since all other form-fields are zero) non-susy $(p-1)$-brane, $(p-2)$-brane, $\ldots$, upto 0-brane. For our purpose of showing the closed string tachyon condensation we do not need these general solutions, but instead we only need the solutions representing charged non-susy $p$-brane intersecting with chargeless non-susy 1-brane and 0-brane. So, we make all the directions in the solutions given in (11) namely, $x^{p}, x^{p-1}, \ldots$, upto $x^{2}$ isotropic by putting $\delta_{3}=\delta_{4}=\cdots=\delta_{p+1}=\delta_{0}$. So the only directions which remain anisotropic are $x^{1}$ and $t$. The solutions (11) then take the form

$$
\begin{aligned}
d s^{2}= & F^{\frac{p+1}{8}}(H \tilde{H})^{\frac{2}{7-p}}\left(\frac{H}{\tilde{H}}\right)^{\frac{p \delta_{1}}{8}+\frac{2(3-p) \bar{\delta}}{(7-p)}}\left(d r^{2}+r^{2} d \Omega_{8-p}^{2}\right) \\
& +F^{-\frac{7-p}{8}}\left(\frac{H}{\tilde{H}}\right)^{\frac{p \delta_{1}}{8}+2 \bar{\delta}}\left(-d t^{2}\right)+F^{-\frac{7-p}{8}}\left(\frac{H}{\tilde{H}}\right)^{\frac{(p-8) \delta_{1}}{8}+2 \bar{\delta}-2 \delta_{2}}\left(d x^{1}\right)^{2} \\
& +F^{-\frac{7-p}{8}}\left(\frac{H}{\tilde{H}}\right)^{\frac{(p-8) \delta_{1}}{8}+2 \bar{\delta}-2 \delta_{0}} \sum_{i=2}^{p}\left(d x^{i}\right)^{2} \\
e^{2 \phi}= & F^{\frac{3-p}{2}}\left(\frac{H}{\tilde{H}}\right)^{\frac{\delta_{1}}{2}(4-p)-8 \bar{\delta}} \\
F_{[8-p]}= & \hat{Q} \operatorname{Vol}\left(\Omega_{8-p}\right)
\end{aligned}
$$

with the parameter relations (3) now changed as

$$
\begin{aligned}
& \alpha-\beta=-\frac{3}{2} \delta_{1} \\
& \frac{1}{2} \delta_{1}^{2}+\frac{1}{2} \alpha\left(\alpha+\frac{3}{2} \delta_{1}\right)+\frac{(p-1)\left[2 \delta_{2}+(p-2) \delta_{0}\right] \delta_{0}}{7-p}=\left(1-\delta_{2}^{2}-(p-1) \delta_{0}^{2}\right) \frac{8-p}{7-p} \\
& \hat{Q}=(7-p) \omega^{7-p}(\alpha+\beta) \sinh 2 \theta
\end{aligned}
$$


Note that in (44) we have for convenience defined

$$
\bar{\delta}=\frac{1}{4} \sum_{i=1}^{p} \delta_{i+1}=\frac{1}{4} \delta_{2}+\frac{(p-1)}{4} \delta_{0}
$$

Also note that the above solution is valid for $1 \leq p \leq 6$. However, for $p=1$ there is no $\delta_{0}$ and the solutions are characterized in that case by four independent parameters namely, $\delta_{1}, \delta_{2}, \omega$ and $\theta$. On the other hand, for $p>1$, the solutions are characterized by five independent parameters namely, $\delta_{0}, \delta_{1}, \delta_{2}, \omega$ and $\theta$. Also from the metric in (4) it is clear that they represent charged non-susy $p$-brane intersecting with chargeless non-susy 1-brane and 0-brane. The $p$-brane here is magnetically charged and the corresponding electrically charged solutions can be obtained by replacing $F_{[8-p]}$ with $F_{[p+2]}=e^{(3-p) \phi / 2} *$ $F_{[8-p]}=(1 / 2) \sinh 2 \theta d(C / F) \wedge d t \wedge d x^{1} \wedge \cdots \wedge d x^{p}$ where $C=(H / \tilde{H})^{\alpha}-(\tilde{H} / H)^{\beta}$. Now after making a coordinate transformation from $r$ to $\rho$ as $r=\rho\left(\frac{1+\sqrt{f}}{2}\right)^{\frac{2}{7-p}}$ where $f=1-\frac{4 \omega^{7-p}}{\rho^{7-p}} \equiv 1-\frac{\rho_{0}^{7-p}}{\rho^{7-p}}$, we can rewrite (44) as,

$$
\begin{aligned}
& d s^{2}=G^{\frac{p+1}{8}} f^{-\frac{\alpha(p+1)}{16}-\frac{p \delta_{1}}{16}-\frac{(3-p) \bar{\delta}}{(7-p)}+\frac{1}{7-p}}\left(\frac{d \rho^{2}}{f}+\rho^{2} d \Omega_{8-p}^{2}\right) \\
& +G^{-\frac{7-p}{8}} f^{\frac{\alpha(7-p)}{16}-\frac{p \delta_{1}}{16}-\bar{\delta}}\left(-d t^{2}\right)+G^{-\frac{7-p}{8}} f^{\frac{\alpha(7-p)}{16}-\frac{(p-8) \delta_{1}}{16}-\bar{\delta}+\delta_{2}}\left(d x^{1}\right)^{2} \\
& +G^{-\frac{7-p}{8}} f^{\frac{\alpha(7-p)}{16}-\frac{(p-8) \delta_{1}}{16}-\bar{\delta}+\delta_{0}} \sum_{i=2}^{p}\left(d x^{i}\right)^{2} \\
& e^{2 \phi}=G^{\frac{3-p}{2}} f^{-\frac{\alpha(3-p)}{4}-\frac{\delta_{1}}{4}(4-p)+4 \bar{\delta}} \\
& F_{[8-p]}=\hat{Q} \operatorname{Vol}\left(\Omega_{8-p}\right)
\end{aligned}
$$

where in (7) we have defined $G(\rho)=\cosh ^{2} \theta-f^{\frac{\alpha+\beta}{2}} \sinh ^{2} \theta$. Note that in general the solution is well defined only for $\rho \geq \rho_{0}$ and $\alpha+\beta \geq 0$ but for the case of black $p$-branes for which $\alpha+\beta=2, \rho$ can be extended to $\rho \geq 0$. The parameter relations remain exactly as in (5). We remark here that the $p$-branes in the above intersecting solutions (7) or (4) are actually the non-susy $\mathrm{D} p$-branes, but we can also write the solutions representing charged non-susy F-string intersecting with chargeless non-susy 0-brane and also charged non-susy NS5-brane intersecting with chargeless non-susy 1-brane and 0-brane by S-dualizing these solutions for $p=1$ and $p=5$ cases respectively. The following argument goes through for these solutions also.

Now it can be easily checked as was also shown in ref.[1] that when

$$
\delta_{1}=-\frac{12}{7}, \quad \delta_{0}=\delta_{2}=-\frac{1}{7}
$$


which implies from (5) and (16)

$$
\alpha=\frac{16}{7}, \quad \beta=-\frac{2}{7}, \quad \text { and } \quad \bar{\delta}=-\frac{p}{28}
$$

then the solutions (77) reduce to in the string frame 11

$$
\begin{aligned}
& d s^{2}=\bar{H}^{\frac{1}{2}}\left(\frac{d \rho^{2}}{f}+\rho^{2} d \Omega_{8-p}^{2}\right)+\bar{H}^{-\frac{1}{2}}\left(-f d t^{2}+\sum_{i=1}^{p}\left(d x^{i}\right)^{2}\right) \\
& e^{2 \phi}=\bar{H}^{\frac{3-p}{2}}, \quad F_{[8-p]}=\hat{Q} \operatorname{Vol}\left(\Omega_{8-p}\right)
\end{aligned}
$$

where $\bar{H}=1+\sinh ^{2} \theta \rho_{0}^{7-p} / \rho^{7-p}$ and $f=1-\rho_{0}^{7-p} / \rho^{7-p}$. This is the standard black D p-brane solutions [10, 11].

Now, if on the other hand, we choose

$$
\delta_{0}=-\frac{2}{7}, \quad \delta_{1}=\frac{4}{7} \quad \text { and } \quad \delta_{2}=\frac{5}{7}
$$

which implies from (15) and (6)

$$
\alpha=\frac{4}{7}, \quad \beta=\frac{10}{7} \quad \text { and } \quad \bar{\delta}=-\frac{2 p-7}{28}
$$

then the solutions (7) reduce to again in the string frame as,

$$
\begin{aligned}
& d s^{2}=\bar{H}^{\frac{1}{2}}\left(\frac{d \rho^{2}}{f}+\rho^{2} d \Omega_{8-p}^{2}\right)+\bar{H}^{-\frac{1}{2}}\left(-d t^{2}+f\left(d x^{1}\right)^{2}+\sum_{i=2}^{p}\left(d x^{i}\right)^{2}\right) \\
& e^{2 \phi}=\bar{H}^{\frac{3-p}{2}}, \quad F_{[8-p]}=\hat{Q} \operatorname{Vol}\left(\Omega_{8-p}\right)
\end{aligned}
$$

These are precisely the KK bubble solutions which can be obtained from the black $p$ brane solutions by making a double Wick rotation on the coordinates $x^{1}$ and $t$. But note that here our solutions (7) continuously interpolate between the black D p-branes (10) and KK-bubbles (13) through a series of classical solutions and there is no need to take any Wick rotation. Note that in order to avoid any conical singularity in (13) at $\rho=\rho_{0}$ the circle $x^{1}$ must have a periodicity of

$$
L=\left(4 \pi \rho_{0} \cosh \theta\right) /(7-p)
$$

Thus we see that (7) in fact represents the interpolating solution from black $p$-brane to the KK BON when the parameters characterizing the solution vary from one set of values

\footnotetext{
${ }^{11}$ Note that the metric in (7) is given in the Einstein frame.
} 
(8) to another (11) 12. So, one might be tempted to think that this continuous change of parameters can be physically interpreted as the transition from the black $p$-brane to static KK BON in parameter space. However, in order to have this possibility, we first need to make sure that the static KK BON are stable at least perturbatively. For this, we perform a time symmetric general bubble initial data analysis which we discuss in the next section.

\section{$3 \quad$ Initial data analysis}

In order to understand the physical meaning of the interpolation, as a first step, we must show that the final bubble configuration is stable so that it does not decay perturbatively further. For this purpose, we consider the general bubble solution and perform the initial data analysis. We take the time symmetric initial data as usual and consider ten dimensional charged bubble solution with non-zero dilaton. Let us consider the spatial metric to have the form,

$$
d s^{2}=\bar{H}(\rho)^{-\frac{7-p}{8}}\left(f(\rho)\left(d x^{1}\right)^{2}+\sum_{i=2}^{p}\left(d x^{i}\right)^{2}\right)+\bar{H}(\rho)^{\frac{p+1}{8}}\left(\frac{d \rho^{2}}{f(\rho) h(\rho)}+\rho^{2} d \Omega_{8-p}^{2}\right)
$$

where $\bar{H}(\rho)$ and $f(\rho)$ are as defined before and $h(\rho)$ will be determined from the constraint equations. Note that the metric is in the Einstein frame. The other fields are given as,

$$
e^{2 \phi}=\bar{H}(\rho)^{\frac{3-p}{2}}, \quad F_{[8-p]}=\hat{Q} \operatorname{Vol}\left(\Omega_{8-p}\right)
$$

For the above time symmetric initial data, the only constraint 13 that needs to be satisfied is

$$
{ }^{9} R=\frac{1}{2}\left(e^{\frac{p-3}{2} \phi} F^{2}+\partial_{\rho} \phi \partial^{\rho} \phi\right)
$$

Using (15) and (16), we solve the constraint equation (17) to obtain,

$$
h(\rho)=1+\frac{\lambda\left(\rho^{7-p}+\rho_{0}^{7-p} \sinh ^{2} \theta\right)}{2(8-p) \rho^{2(7-p)}-(9-p) \rho^{7-p} \rho_{0}^{7-p}+\left[(9-p) \rho^{7-p}-2 \rho_{0}^{7-p}\right] \rho_{0}^{7-p} \sinh ^{2} \theta}
$$

\footnotetext{
${ }^{12}$ Here we point out that in order to give a physical interpretation of this interpolating solution as the transition caused by the closed string tachyon condensation, as we will discuss in section 4 , all the brane directions (not just $x^{1}$ ) would have to be compactified with the asymptotic sizes of the circles associated with various brane directions of the same order as $L$. Note also that the parameters $\theta$ and $\rho_{0}$ change during the transition.

${ }^{13}$ We don't have additional non-trivial constraints for $F$ and $\phi$.
} 
where $\lambda$ is an integration constant. This therefore gives a three-parameter $\left(\lambda, \rho_{0}\right.$ and $\left.\theta\right)$ family of time symmetric, asymptotically flat initial data. Note that when we put $\theta=0$, the charge parameter $\hat{Q}$ as well as the dilaton vanishes and in that case $h(\rho)$ becomes

$$
h(\rho)=1+\frac{\lambda}{2(8-p) \rho^{7-p}-(9-p) \rho_{0}^{7-p}}
$$

For $p=5$, this matches exactly with eq.(3.13) of [4] as expected. In order to avoid conical singularity at $\rho=\rho_{0}$, the circle $x^{1}$ must have a periodicity

$$
\begin{aligned}
L & =\frac{4 \pi \rho_{0} \cosh \theta}{7-p}\left(1+\frac{\lambda}{(7-p) \rho_{0}^{7-p}}\right)^{-\frac{1}{2}} \\
& =\frac{4 \pi \hat{\rho}_{0}^{\frac{7-p}{2}}}{7-p}\left(\hat{\rho}_{0}^{7-p}-\frac{\hat{Q}^{2}}{(7-p)^{2} \hat{\rho}_{0}^{7-p}}\right)^{-\frac{5-p}{2(7-p)}}\left[1+\frac{\lambda}{(7-p)\left(\hat{\rho}_{0}^{7-p}-\frac{\hat{Q}^{2}}{(7-p)^{2} \hat{\rho}_{0}^{\gamma-p}}\right)}\right]^{-1 / 2}
\end{aligned}
$$

where in the second equality we have defined $\hat{\rho}_{0}^{7-p}=\rho_{0}^{7-p} \cosh ^{2} \theta$ and used the charge expression $14 \hat{Q}=(7-p) \rho_{0}^{7-p} \sinh \theta \cosh \theta$ for the purpose of later comparison. The ADM mass of these bubbles can be obtained from the metric (15) as,

$$
M=\frac{\Omega_{8-p}}{2 \kappa^{2}}\left[\frac{(5-p) \hat{Q}^{2}}{2(7-p)^{2} \hat{\rho}_{0}^{7-p}}+\frac{9-p}{2} \hat{\rho}_{0}^{7-p}-\frac{(4 \pi)^{2} \hat{\rho}_{0}^{7-p}}{2(7-p) L^{2}}\left(\hat{\rho}_{0}^{7-p}-\frac{\hat{Q}^{2}}{(7-p)^{2} \hat{\rho}_{0}^{7-p}}\right)^{\frac{2}{7-p}}\right]
$$

where $\Omega_{n}=2 \pi^{(n+1) / 2} / \Gamma((n+1) / 2)$ is the volume of an $n$-dimensional unit sphere and $2 \kappa^{2}=16 \pi G$, with $G$, the Newton's constant. The mass has been calculated from the metric (15) and we have eliminated the unknown integration constant $\lambda$ in that expression using (20) as well as the following relation between $\rho_{0}$ and $\hat{\rho}_{0}$,

$$
\rho_{0}^{7-p}=\hat{\rho}_{0}^{7-p}\left(1-\frac{\hat{Q}^{2}}{(7-p)^{2} \hat{\rho}_{0}^{2(7-p)}}\right)
$$

Eq.(22) clearly tells us that $15 \hat{\rho}_{0}^{7-p} \geq \hat{Q} /(7-p)$. Note that the mass in (21) takes a positive value $M=\frac{\Omega_{8-p}}{2 \kappa^{2}} \hat{Q}$ when $\hat{\rho}_{0}$ takes the lowest value while $M \rightarrow-\infty$ when $\hat{\rho}_{0} \rightarrow \infty$.

\footnotetext{
${ }^{14}$ Without loss of generality, we assume from now on $\hat{Q} \geq 0$ i.e., $\theta \geq 0$. Note that the charge is now a result of the flux on the non-contractible $S^{8-p}$ since there is no longer a singularity to act as the source of the charge.

${ }^{15}$ This can also be seen from the ratio

$$
\frac{(7-p) \hat{\rho}_{0}^{7-p}}{\hat{Q}}=\frac{\cosh \theta}{\sinh \theta} \geq 1 .
$$

If we ignore this constraint, the mass $\rightarrow \infty$ as $\hat{\rho}_{0} \rightarrow 0$, indicating the possibility that the small bubble cannot contract when $\hat{Q} \neq 0$. Indeed if we consider a 4-parameter family of initial data as we will mention later on, there will be no such constraint since in that case the $\hat{Q}$ is independent of $\rho_{0}$ and $\theta$.
} 
So, there is no lower bound on mass and the positive energy theorem fails for our initial data as was also found for the case studied in [4].

Thus, for fixed values of $\hat{Q}$ and $L$, the expression for ADM mass (21) has extremum when $d M / d \hat{\rho}_{0}=0$ and this implies,

$$
L=\frac{4 \pi \hat{\rho}_{0}^{\frac{7-p}{2}}}{7-p}\left(\hat{\rho}_{0}^{7-p}-\frac{\hat{Q}^{2}}{(7-p)^{2} \hat{\rho}_{0}^{7-p}}\right)^{-\frac{5-p}{2(7-p)}}
$$

In comparison with the second expression in (20), we find that the extremum occurs at $\lambda=0$ (or, $h(\rho)=1)$. The resulting metric in (15) is now the spatial part of the static bubble one obtained from the double Wick rotations of black p-brane in Einstein frame.

We now come to examine whether the extremum is a local minimum or a maximum. This will give us indications about the nature of stability of the static bubbles under consideration. For this, let us consider $\hat{Q}=0$ and $\hat{Q} \neq 0$ separately. When $\hat{Q}=0$, i.e., $\theta=0$, we have from (24)

$$
\hat{\rho}_{0}=\rho_{0}=\frac{7-p}{4 \pi} L .
$$

In other words, the static bubble size is always less than $L$, the circle size at infinity. One can easily check that the extremum is now a maximum, as expected, by calculating $\frac{d^{2} M}{d \hat{\rho}_{0}^{2}}<0$ at this $\hat{\rho}_{0}$. So this is an unstable static bubble. This also implies the usual intuition that bubbles which are smaller than the size of the circle at infinity contract while larger ones expand as discussed in [4. Adding flux (i.e., for non-vanishing charge $\hat{Q} \neq 0$ ) on the sphere causes it to expand and the vacuum bubbles which would normally contract could become stable static bubbles with this flux. So let us turn our attention to this case next.

For this, let us first determine the number of solutions for $\hat{\rho}_{0}$ from (24) for fixed values of $\hat{Q} \neq 0$ and $L$. This number is actually the same as that for $\theta$ for a given ratio of $C=\hat{Q} / L^{7-p}$ determined from the following equation

$$
\frac{\hat{Q}}{L^{7-p}}=\frac{(7-p)^{8-p}}{(4 \pi)^{7-p}} \frac{\sinh \theta}{\cosh ^{6-p} \theta}=C(\theta) .
$$

In obtaining (26), we have used the expression $\hat{Q}=(7-p) \rho_{0}^{7-p} \sinh \theta \cosh \theta$ and the expression (14) for $L$ for the static bubble which holds also for the extremum as shown above. Once $\theta$ is determined, the $\rho_{0}$ can be determined either from the $\hat{Q}$ expression or from the equation (14) for $L . \hat{\rho}_{0}$ is then determined from $\rho_{0}$. This provides an alternative (also much simpler) way to determine the number of solutions for $\hat{\rho}_{0}$ from (24). In the following, we consider $p<5$ cases first and then the remaining $p=5,6$ cases can be considered in a much direct and simpler way afterwards. 


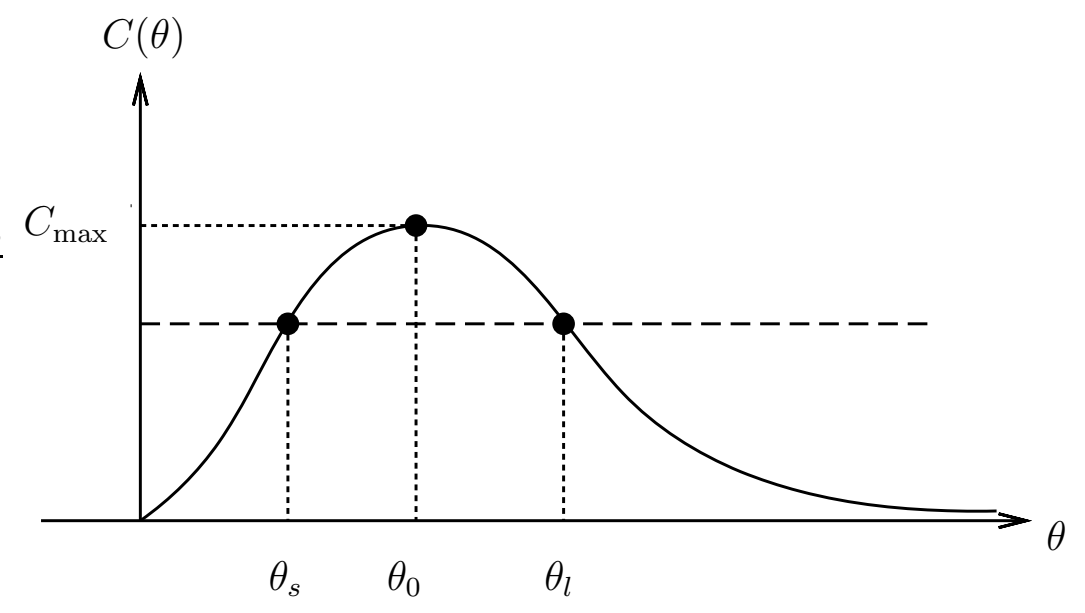

Figure 1: The $\theta_{0}$ gives rise to the maxima of $C(\theta)$, i.e., $C_{\max }$ while $\theta_{s}$ and $\theta_{l}$ are two solutions of (26) when $\hat{Q} / L^{7-p}<C_{\max }$ for $p \leq 4$

It is not difficult to check for $p<5$ that $C(\theta)$ in (26) has a unique maximum at $\sinh ^{2} \theta=1 /(5-p)$. This can also be seen from the fact that $C(\theta)>0$ in general and $C(\theta) \rightarrow 0$ for both $\theta \rightarrow 0$ and $\theta \rightarrow \infty$. This maximum is given as,

$$
C_{\max }=\left(\frac{\hat{Q}}{L^{7-p}}\right)_{\max }=\frac{(7-p)^{8-p}(5-p)^{\frac{5-p}{2}}}{(4 \pi)^{7-p}(6-p)^{\frac{6-p}{2}}}<1 .
$$

So for any given positive ratio $\hat{Q} / L^{7-p}=C(\theta)<C_{\max }$, we will have two solutions for $\theta$ from equation (26) since the constant line $C(\theta)=\hat{Q} / L^{7-p}$ will intercept twice with the curve

$$
C(\theta)=\frac{(7-p)^{8-p}}{(4 \pi)^{7-p}} \frac{\sinh \theta}{\cosh ^{6-p} \theta} .
$$

The various features mentioned here are shown in Fig.1.

Now we can relate $\hat{\rho}_{0}$ with $\theta$ by using (14) and the relation $\hat{\rho}_{0}^{7-p}=\rho_{0}^{7-p} \cosh ^{2} \theta$ as,

$$
\hat{\rho}_{0}=\frac{(7-p) L}{4 \pi} \cosh ^{\frac{p-5}{7-p}} \theta
$$

So, this implies that we have two solutions for $\hat{\rho}_{0}$ from (24) under the same condition, the small $\theta$ corresponds to large $\hat{\rho}_{0}$ and large $\theta$ corresponds to small $\hat{\rho}_{0}$. Since these two solutions are extrema of ADM mass, therefore one extremum must give a maximum and the other gives the minimum. Given the fact that $M \rightarrow-\infty$ as $\hat{\rho}_{0} \rightarrow \infty$, the extremum with the large $\hat{\rho}_{0}$ must give the maximum while the one with the small $\hat{\rho}_{0}$ gives a local minimum. This is entirely consistent with our anticipation from the $\hat{Q}=0$ discussion 


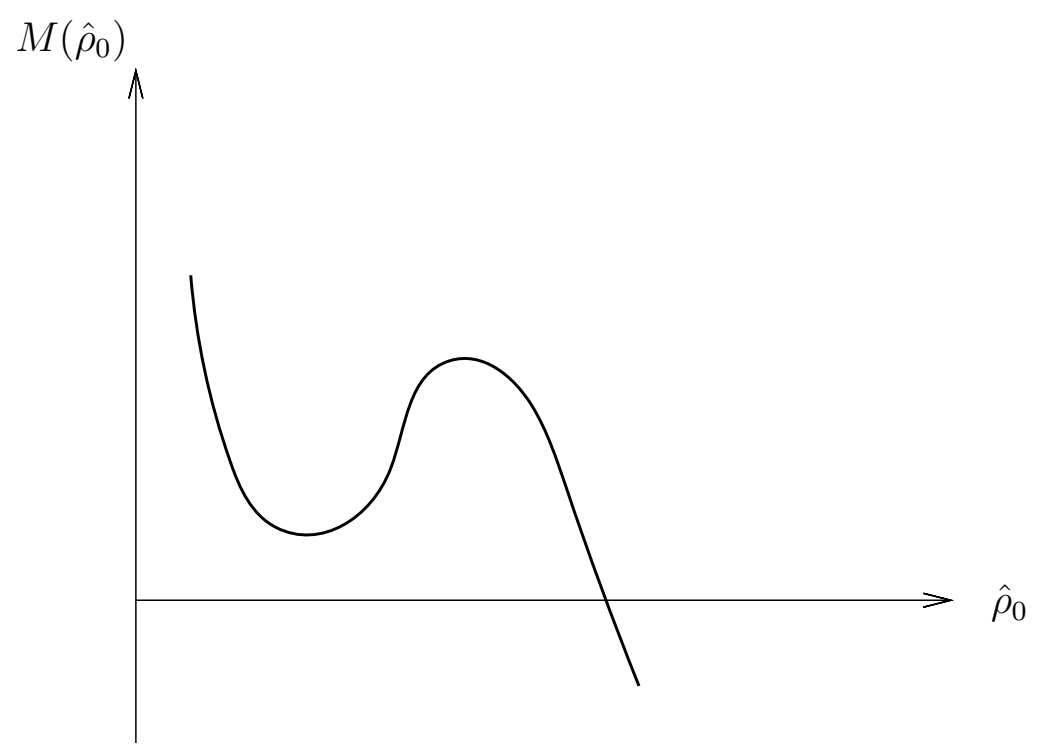

Figure 2: The characteristic behavior of the mass vs. $\hat{\rho}_{0}$ as given in (21) for $p \leq 4$. The local minimum is at the $\hat{\rho}_{0}$ as given by (29) with $\theta=\theta_{l}$ while the local maximum is at the $\hat{\rho}_{0}$ determined by the same equation with now $\theta=\theta_{s}$.

given above. This characteristic feature of the ADM mass versus $\hat{\rho}_{0}$ is shown in Fig.2 for $p \leq 4$. Note that, however, even the local stable static bubble is non-perturbatively unstable since the ADM mass is unbounded from below.

When the ratio $\hat{Q} / L^{7-p}$ takes the maximum value as given in (27), we expect that the two merge into a single extremum with $\frac{d^{2} M}{d \hat{\rho}_{0}^{2}}=0$ when the $\hat{\rho}_{0}$ takes its extremum value. This can be checked independently from the following

$$
\begin{aligned}
\left.\frac{d^{2} M}{d \hat{\rho}_{0}^{2}}\right|_{\hat{\rho}_{0}=c_{0}}= & -\frac{\Omega_{8-p}}{2 \kappa^{2}} \frac{9-p}{7-p} \frac{(4 \pi)^{2} c_{0}^{7-p}}{L^{2}}\left(1-\frac{\hat{Q}^{2}}{(7-p)^{2} c_{0}^{2(7-p)}}\right)^{-\frac{2(6-p)}{7-p}} \\
& \times\left[1-(6-p) \frac{\hat{Q}^{2}}{(7-p)^{2} c_{0}^{2(7-p)}}\right]\left(1-\frac{5-p}{9-p} \frac{\hat{Q}^{2}}{(7-p)^{2} c_{0}^{2(7-p)}}\right)
\end{aligned}
$$

where we have set $\hat{\rho}_{0}=c_{0}$ with $c_{0}$ the solution of the equation (24). By demanding $\left.\frac{d^{2} M}{d \hat{\rho}_{0}^{2}}\right|_{\hat{\rho}_{0}=c_{0}}=0$, we indeed have one unique solution $c_{0}^{7-p}=\sqrt{6-p} \hat{Q} /(7-p)$ which corresponds to the maximum ratio of $\hat{Q} / L^{7-p}$ as anticipated above.

Now we try to find some concrete solutions from (24) under certain special conditions 
to support the above general analysis. For this, we rewrite (24) in the following form,

$$
\frac{(4 \pi)^{2} \hat{\rho}_{0}^{2}}{(7-p)^{2} L^{2}}=\left(1-\frac{\hat{Q}^{2}}{(7-p)^{2} \hat{\rho}_{0}^{2(7-p)}}\right)^{\frac{5-p}{7-p}} .
$$

For $p \leq 4$, we find the following solutions from (31) as

$$
\begin{aligned}
& \text { (i) } \hat{\rho}_{0} \approx \frac{(7-p) L}{4 \pi}, \text { when } \quad \hat{\rho}_{0} \gg\left(\frac{\hat{Q}}{7-p}\right)^{\frac{1}{7-p}} \\
& \text { (ii) } \hat{\rho}_{0} \approx\left(\frac{\hat{Q}}{7-p}\right)^{\frac{1}{7-p}}, \quad \text { when } \quad \hat{\rho}_{0} \ll L
\end{aligned}
$$

So, for case $(i)$, the extremum occurs at $\hat{\rho}_{0} \approx(7-p) L /(4 \pi)$, and for case (ii), the extremum occurs at $\hat{\rho}_{0}^{7-p} \approx \hat{Q} /(7-p)$. Note that in both cases $L^{7-p} \gg \hat{Q}$, satisfying the allowed constraint $L^{7-p}>\hat{Q}$ in (27).

We thus find that for $L^{7-p} \gg \hat{Q}$, we have two static bubbles, one large bubble at $\hat{\rho}_{0} \approx(7-p) L /(4 \pi)$ and one small bubble at $\hat{\rho}_{0}^{7-p} \approx \hat{Q} /(7-p)$. We calculate $d^{2} M / d \hat{\rho}_{0}^{2}$ using (30) at the two values of $\hat{\rho}_{0}$ and find that the large bubble corresponds to a maximum and the small bubble correspond to a minimum. This is entirely consistent with our above general analysis.

Now let us consider $p=5,6$. It is clear from the equation (31) that in both cases there is only one extremum. For $p=5$, the extremum is at $\hat{\rho}_{0}=L /(2 \pi)$ while for $p=6$ it is at $\hat{\rho}_{0}=\sqrt{L^{2} /(4 \pi)^{2}+\hat{Q}^{2}}$. Therefore, considering the bound from (26)), i.e., $L^{2} \geq 2 \pi^{2} \hat{Q}$, the extremum for $p=5$ gives $\hat{\rho}_{0}^{2}=L^{2} /(2 \pi)^{2} \geq \hat{Q} / 2$ which satisfies the constraint $\hat{\rho}_{0}^{7-p} \geq \hat{Q} /(7-p)$. For $p=6$, we don't have any bound from (26) but now the extremum $\hat{\rho}_{0}=\sqrt{L^{2} /(4 \pi)^{2}+\hat{Q}^{2}}$ automatically satisfies the constraint $\hat{\rho}_{0}^{7-p} \geq \hat{Q} /(7-p)$. The existence of a single extremum is also consistent with the fact that there exists a single solution of $\theta$ in (26) for either $p=5$ or $p=6$. This can be understood from that for $p=5$, the $C(\theta)$ has a maximum at $\theta \rightarrow \infty$ while for $p=6, C(\theta)$ increases monotonically to infinity. The corresponding features are shown in Fig.3 for $p=5$ and in Fig.4 for $p=6$, respectively. The extremum for each case is actually a maximum which can be seen from the sign of the double derivative $d^{2} M / d \hat{\rho}_{0}^{2}$ in (30) at the corresponding extremum value of $\hat{\rho}_{0}$. In other words, the bubbles in both cases are static but locally unstable. Note that adding flux on the sphere for $p=5,6$ does not change the qualitative picture from vanishing flux case.

So, the general bubble initial data analysis tells us that for $p \leq 4$, there are two static bubbles if $\hat{Q} / L^{7-p}<C_{\max }$. These bubbles correspond to the doubly Wick rotated form 


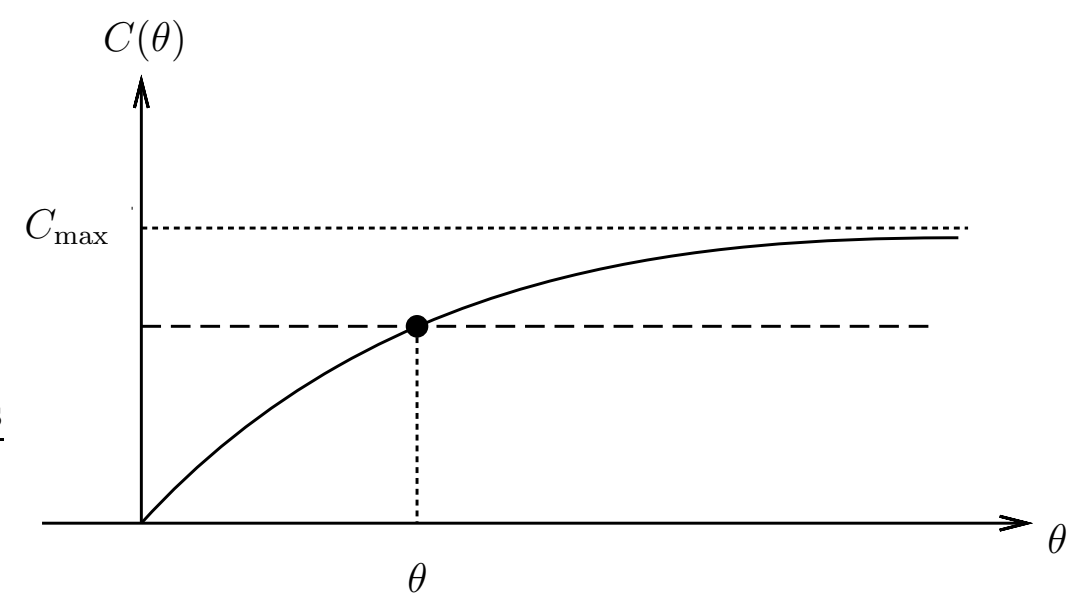

Figure 3: The black dot represents a solution of (26) with fixed ratio $\hat{Q} / L^{2}<1 /\left(2 \pi^{2}\right)$ for $p=5$. The corresponding $\theta$ gives a static but unstable bubble.

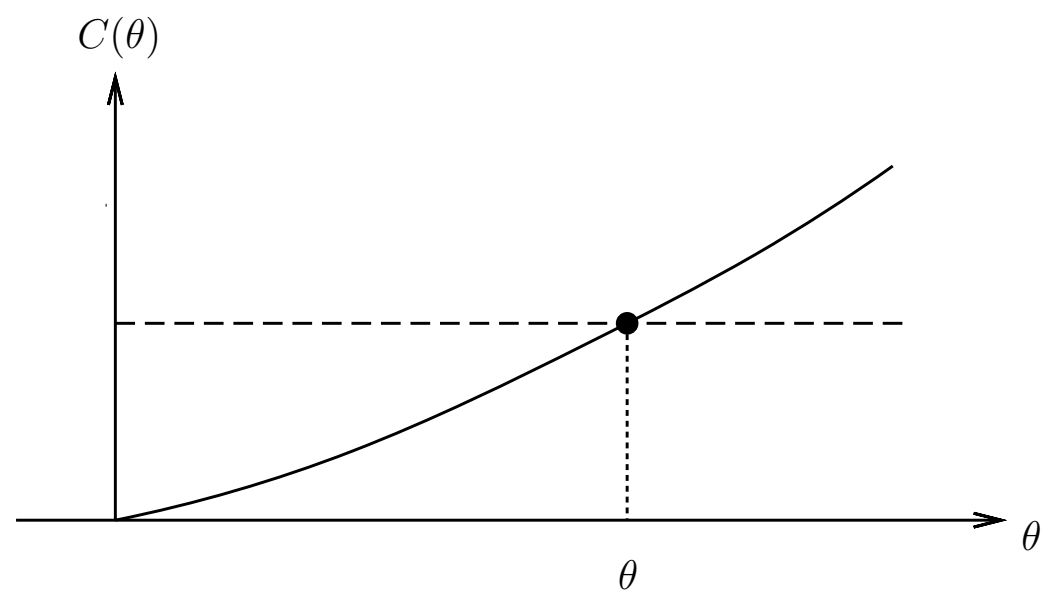

Figure 4: The black dot represents a solution of (26) with any given ratio $\hat{Q} / L$ for $p=6$. The corresponding $\theta$ gives again a static but unstable bubble. 
of the black $p$-branes and of them the smaller bubble is locally stable and the larger one is locally unstable. On the other hand for $p=5,6$, there is an unstable static bubble but for $p=5$ an additional condition $L^{2}>2 \pi^{2} \hat{Q}$ needs to be satisfied.

From this analysis, since we found that only for $p \leq 4$ and for $\hat{Q} / L^{7-p}<C_{\max }$, there can be a static (locally) stable bubble, therefore, only in those cases the interpolating solution (7) have the possibility to be interpreted as the transition from black D $p$-brane to static KK BON. For all other cases, the black D p-branes would decay into dynamical bubbles. We also performed a 4-parameter family of time-symmetric, asymptotically flat initial data analysis in which the charge parameter $\hat{Q}$ is treated to be independent of $\rho_{0}$ and $\theta$ and the same conclusion is reached. Therefore, the above picture is believed to be quite generic. In the following, we will provide another independent check of the nature of stability of the static charged bubbles we obtained.

For this, let us recall the discussion given in [4] by Horowitz following [16]. It has been argued there that for black strings and the corresponding static bubbles, the perturbative stability of bubbles is directly related to that of the black strings since the two are related to each other by double Wick rotation. The study of perturbations of black strings and $p$-branes started with the work of Gregory and Laflamme [17]. It is known that the short wavelength modes are always stable but the long wavelength modes are sometimes unstable. The instability of long wavelength modes further implies a static perturbation and the existence of a static perturbation in turn usually indicates an instability. The Gubser-Mitra conjecture [18] states that charged black strings will be unstable if and only if their specific heat is negative. Based on the above, Horowitz concluded that for spacetime dimensions $D=5,6$ and large $L$, there is always a static perturbation of the black string, therefore an unstable perturbation of the corresponding bubble. In higher dimensions, the static bubbles are perturbatively stable or unstable depending on how close to extremality the corresponding black string is.

Since black $p$-branes (for $p \leq 6$ ) in $D=10$ are related to black strings in $D=$ $11-p$ through the so-called double dimensional reduction (here dimensional reductions are performed simultaneously on both spacetime and brane spatial directions), for example, black 6-branes and 5-branes in $\mathrm{D}=10$ are related to black strings in $\mathrm{D}=5$ and 6 respectively, so from the above discussion, we must have black 6-branes and 5-branes in $\mathrm{D}=10$ unstable and therefore the corresponding static bubbles are also unstable. On the other hand, since black $p$-branes with $p \leq 4$ are related to black strings in $D=11-p>6$, so the perturbative stability of black $p$-branes with $p \leq 4$, should depend on how close they are to their respective extremality and this also determines the stability of the corresponding bubbles. We can understand the above from the signs of the specific heat 
of the black $p$-branes as follows. The specific heat can be calculated once a black $p$-brane configuration (such as (10) ) is given, for example, see [19], and its sign is determined by the sign of the following quantity

$$
\rho_{0}^{7-p}\left[(6-p) \sinh ^{2} \theta-\cosh ^{2} \theta\right]
$$

It is easy to see that for $p=5,6$, its sign is always negative, signalling the instability as

described above. For $p \leq 4$, its sign indeed depends on how close the black $p$-branes are to the extremality which corresponds to taking $\theta \rightarrow \infty$. As will be discussed in the following section, the closed string tachyon condensation occurring on or outside the horizon always requires large $\theta$ for large $L$, so to leading order (33) can be written as,

$$
\frac{(5-p)}{4} \rho_{0}^{7-p} e^{2 \theta}>0
$$

for $p \leq 4$, and therefore the corresponding static bubbles are classically stable. This completely independent analysis supports the results of our initial data analysis.

\section{Physical interpretation of the interpolation}

Let us next try to understand the underlying mechanism for the transition. The existence of the interpolation indicates a possible transition from black $\mathrm{D} p$-brane to the static and locally stable KK BON for $p \leq 4$ (the interpolation makes sense only for these cases). The closed string tachyon condensation could be the possible mechanism for these cases if the curvature on the horizon (where the tachyon condensation takes place) is much smaller than the string scale, otherwise we will reach the correspondence point where there is no brane description and the black D-brane would make transition to the open string modes [4]. For $p>4$, we have seen that there exist static bubbles, but the bubbles in these cases are unstable and the corresponding interpolation cannot hold even true classically since the static bubble will contract or expand under any small perturbation even though the interpolating solutions exist. We expect that the closed string tachyon condensation in these cases if possible must give rise to the corresponding dynamical bubbles as we will see.

Now from the metric given in (10) we see that if the closed string tachyon condensation occur on the horizon, then the size of the circle associated with the coordinate $x^{1}$ is

$$
L=l_{s} \cosh ^{\frac{1}{2}} \theta
$$


where $l_{s}$ is the fundamental string length. Also note that since $L \gg l_{s}$, the angle $\theta$ must be very $\operatorname{larg} 16$ and therefore $L$ can be approximated as

$$
L=l_{s} \cosh ^{\frac{1}{2}} \theta \approx \frac{l_{s}}{\sqrt{2}} e^{\theta / 2}
$$

The charge of the black D $p$-branes can also be obtained from the form-field given in (10) as

$$
\hat{Q}=(7-p) \rho_{0}^{7-p} \sinh \theta \cosh \theta \approx \frac{7-p}{4} \rho_{0}^{7-p} e^{2 \theta}
$$

Also the size of the horizon can be obtained from the metric in (10) as,

$$
Z=\rho_{0} \cosh ^{\frac{1}{2}} \theta \approx \frac{\rho_{0}}{\sqrt{2}} e^{\theta / 2}
$$

Since the curvature at the horizon has to be much smaller compared to the string scale, so we get,

$$
\mathcal{R} \sim \frac{1}{Z^{2}} \ll \frac{1}{l_{s}^{2}} \Rightarrow \frac{\rho_{0}}{\sqrt{2} l_{s}} \gg e^{-\theta / 2}
$$

Now we assume that the underlying mechanism for the transition from black $p$-branes to bubbles is via closed string tachyon condensation, the final bubbles can be either (stable or unstable) static or dynamical. From our initial data analysis in section 3 , we know that we can have stable static bubbles only for $p \leq 4$ and for $p>4$ there exist only unstable static bubbles. So, we discuss the possible transitions for $p \leq 4$ and $p>4$ separately. Also for simplicity we will assume that the closed string tachyon condensation occurs on the horizon. As will be discussed that there exist situations for which the parameters characterizing the bubbles can be different from those of the black $p$-branes, so we denote the bubble parameters with a 'tilde'. From (13) we give below the flux $\left(Q_{b}\right)$ associated with the bubble, the size $\left(Z_{b}\right)$ of the bubble and the circle size $\left(L_{b}\right.$, see eq. (14) where we rename $L$ as $L_{b}$ ) of the circle at infinity as,

$$
\begin{aligned}
Q_{b} & =(7-p) \tilde{\rho}_{0}^{7-p} \sinh \tilde{\theta} \cosh \tilde{\theta} \\
Z_{b} & =\tilde{\rho}_{0} \cosh ^{\frac{1}{2}} \tilde{\theta} \\
L_{b} & =\frac{4 \pi}{7-p} \tilde{\rho}_{0} \cosh \tilde{\theta}
\end{aligned}
$$

We have seen in section 3 that there exist two static bubbles with small one stable and the large one unstable for $p \leq 4$ and these two static bubbles occur at two values of $\tilde{\theta}$ corresponding to the two points where the constant line $C(\tilde{\theta})=\hat{Q}_{b} / L_{b}^{7-p}$ meets the curve

\footnotetext{
${ }^{16}$ Therefore, the black $p$-branes must be nearly extremal which guarantees the perturbative stability of static bubbles for $p \leq 4$ as mentioned in the previous section.
} 
(28). The curve (28) has a maximum at $\sinh \tilde{\theta}_{0}=1 / \sqrt{5-p}<1$ and so, $\tilde{\theta}_{0}<1$. The two values of $\tilde{\theta}$ at which the static bubbles exist may be denoted as $\tilde{\theta}_{s}$ and $\tilde{\theta}_{\ell}$ where $\tilde{\theta}_{s}<\tilde{\theta}_{0}<\tilde{\theta}_{\ell}$. Let us first take $\tilde{\theta}_{s}$ as the static bubble parameter, then since this is a very small angle $\tilde{\theta}_{s}<\tilde{\theta}_{0}<1$, (40) can be approximated, to leading order, as

$$
\begin{aligned}
Q_{b} & =(7-p) \tilde{\rho}_{0}^{7-p} \tilde{\theta}_{s} \\
Z_{b} & =\tilde{\rho}_{0} \\
L_{b} & =\frac{4 \pi}{7-p} \tilde{\rho}_{0}
\end{aligned}
$$

If the black brane makes a transition to a static bubble through closed string tachyon condensation, the charge must be conserved. Also the horizon size of the black brane must be equal to the bubble size and the size of the compact circle at infinity must be the same since the local closed string tachyon condensation should not have much effects on them.

At this point we would like to clarify why we don't identify the other brane directions (other than $x^{1}$ ) as well as the dilaton profile before and after the closed string tachyon condensation, i.e., for the black $p$-brane and the BON. It is clear from (10) and (13) that if we do that then the harmonic functions would have to be identified and this would imply that the parameters of the two solutions would have to be identified and therefore the curvature at the horizon would always be of the order of string scale and the supergravity description would break down. In other words, with the identifications of the other directions along the original black branes with those of the corresponding bubble, the black branes can not decay into static BON, but can decay only into dynamical BON. It is true that this situation can not be avoided as long as the other brane directions remain non-compact. (Here we remark paranthetically that even for the case of black D3-brane considered in [4], the above comments remain valid and in fact there can not be a transition from black D3-brane to static BON as claimed otherwise in [4].) However, we can get around this problem if we compactify all the spatial directions of the brane with their asymptotic sizes having the same order of magnitude as $L$ (say). Now it is clear that if the closed string tachyon condensation occurs on the horizon then the sizes of all the brane directions would be of the order of string length there. So the fundamental string can wrap around any of the spatial directions to give rise to closed string tachyon and for this reason we should not identify the directions along the original brane before and after the closed string tachyon condensation. However, the various spatial directions along the brane should still be identified asymptotically where the tachyon condensation has no effect at all since the tachyon condensation takes an infinite amount of coordinate 
time to complete due to the horizon red-shift factor and its effect takes an infinite amount of coordinate time to reach this region 17 . This is precisely the reason that asymptotically the two configurations (10) and (13) are identical. As for the transverse sphere, i.e., the horizon and the size of the bubble, since they are non-contractible and have to be of large size with the physical radius much larger than the string scale $\left(l_{s}\right)$ for the supergravity description to remain valid (therefore the string cannot wrap on this sphere to give rise to tachyon condensation), we then expect their sizes to remain unaffected before and after the closed string tachyon condensation occurring along the brane directions. This is the reason we have identified the size of the horizon with the bubble size. Given the dilaton as the effective string coupling and the closed string tachyon condensation as a perturbative process, the dilaton must remain small for the occurrence of this process. The transition from black brane to BON by closed string tachyon condensation is a perturbative process and the dilaton remains small during the transition. So, the dilaton can not be thought of as an additional spatial direction (as we do when we uplift the string theory solution to higher dimensions for strong coupling, therefore large dimensional size) for which one might think that it should be identified before an after the tachyon condensation. Now since we are dealing with two different solutions (black brane and bubble before and after the tachyon condensation), there is no reason why the dilaton profile should be identified on the horizon and this is also consistent with the non-identification of the brane directions discussed above. For definiteness and for the purpose of modeling this process, in what follows, we choose one direction, namely $x^{1}$, to be the direction along which closed string tachyon condensation takes place but we need to keep in mind that the tachyon condensation can occur along any spatial direction of the brane.

Going by the above arguments, we equate (37), (38) and (36) with the corresponding

\footnotetext{
${ }^{17}$ Although it is known $[9$ that the local proper time for the completion of the closed string tachyon condensation on the horizon is of the order of string scale, however, precisely for this reason, the tachyon condensation process viewed by an observer at infinity (with respect to whom the ADM mass is measured) is a very slow process and therefore to this observer the tachyon condensation can be taken as an adiabatic process. This is consistent with the initial data analysis performed in the previous section since it is based on the ADM mass which is measured by the asymptotic observer and also with our interpolating solution as we will explain.
} 
quantities in (41) to get,

$$
\begin{aligned}
\tilde{\rho}_{0}^{7-p} \tilde{\theta}_{s} & =\frac{1}{4} \rho_{0}^{7-p} e^{2 \theta} \\
\tilde{\rho}_{0} & =\frac{1}{\sqrt{2}} \rho_{0} e^{\theta / 2} \\
\tilde{\rho}_{0} & =\frac{4 \pi}{\sqrt{2}(7-p)} l_{s} e^{\theta / 2}
\end{aligned}
$$

From (42) we get,

$$
\rho_{0}=\frac{4 \pi}{7-p} l_{s}, \quad \tilde{\theta}_{s} \sim e^{(p-3) \theta / 2}
$$

Note from (39) that with this $\rho_{0}$, the curvature remains small compared to string scale since $\theta$ is very large. Also since $\tilde{\theta}_{s}$ is very small, the second condition in (43) is consistent as long as $p<3$. Now since the small $\tilde{\theta}$ corresponds to large bubble which is static but unstable, so, for $p<3$, the black branes will make transitions to unstable static bubble by closed string tachyon condensation. However, since the bubble is unstable, under slight perturbation, the bubble can either expand further to infinity or it can contract to eventually settle down to smaller stable static bubble. This way, black D1 and D2 branes can indeed make a transition to stable static bubble by closed string tachyon condensation and under these conditions the interpolations can have physical interpretation as the closed string tachyon condensation.

Let us next take $\tilde{\theta}_{\ell}$ as the static bubble parameter, where the angle can be large (since we have already considered the case of small angles and the conditions can be met only for $p<3$ as we have just seen). Now (40) can be approximated, to leading order, as,

$$
\begin{aligned}
Q_{b} & =\frac{7-p}{4} \tilde{\rho}_{0}^{7-p} e^{2 \tilde{\theta}_{\ell}} \\
Z_{b} & =\frac{1}{\sqrt{2}} \tilde{\rho}_{0} e^{\tilde{\theta}_{\ell} / 2} \\
L_{b} & =\frac{4 \pi}{7-p} \tilde{\rho}_{0} e^{\tilde{\theta}_{\ell}}
\end{aligned}
$$

Now equating (44) with (37), (38) and (36) we get,

$$
\tilde{\rho}_{0}=\rho_{0} e^{\frac{\theta-\tilde{\theta}_{\ell}}{2}}, \quad \tilde{\rho}_{0}=\rho_{0} e^{\frac{2\left(\theta-\tilde{\theta}_{\ell}\right)}{7-p}}, \quad \rho_{0}=\frac{(7-p) l_{s}}{4 \sqrt{2} \pi} e^{-\tilde{\theta}_{\ell} / 2}
$$

Note that the first two relations in (45) can be made consistent only for $p=3$. The last condition when compared with (39) can be consistent only if $\theta \gg \tilde{\theta}_{\ell}$. Since $\tilde{\theta}_{\ell}$ corresponds to small bubble which is static as well as stable, so, in this case the black D3-brane makes a 
transition directly to the static stable bubble through closed string tachyon condensation and the interpolation has a physical interpretation.

For $p=4$, it is clear from our above analysis that black D4-brane can not make a transition either to a stable static bubble or to an unstable static bubble via closed string tachyon condensation directly. It can only first make a transition to a dynamical bubble. Then depending on the size of the dynamical bubble created initially in comparison with that of the stable or unstable static bubble for a given ratio $\hat{Q} / L^{3}<C_{\max }$, we can have the dynamical bubble to expand to infinity if the size is larger than the size of the static unstable bubble or otherwise to settle down to the static stable bubble. For this, let us first try to find out the sizes of the stable static bubble and unstable static bubble, respectively, for $p=4$ case. The equation determining $\tilde{\theta}_{s}$ and $\tilde{\theta}_{l}$ for given $\hat{Q}=\hat{Q}_{b}$ and $L=L_{b}$ is (see eq.(26) $)$,

$$
\begin{aligned}
& \frac{\hat{Q}^{2}}{L^{6}}=\frac{3^{8}}{(4 \pi)^{6}} \frac{\cosh ^{2} \tilde{\theta}-1}{\cosh ^{4} \tilde{\theta}} \\
& \Rightarrow \cosh ^{4} \tilde{\theta}-4\left(\frac{C_{\max } L^{3}}{\hat{Q}}\right)^{2} \cosh ^{2} \tilde{\theta}+4\left(\frac{C_{\max } L^{3}}{\hat{Q}}\right)^{2}=0
\end{aligned}
$$

where we have used $C_{\max }=3^{4} /\left[2(4 \pi)^{3}\right]$ from (27). The solutions to the above equation are given as,

$$
\begin{aligned}
& \cosh \tilde{\theta}_{s}=\sqrt{2}\left(\frac{C_{\max } L^{3}}{\hat{Q}}\right)\left[1-\sqrt{1-\frac{1}{\left(\frac{C_{\max } L^{3}}{\hat{Q}}\right)^{2}}}\right]^{\frac{1}{2}} \\
& \cosh \tilde{\theta}_{\ell}=\sqrt{2}\left(\frac{C_{\max } L^{3}}{\hat{Q}}\right)\left[1+\sqrt{1-\frac{1}{\left(\frac{C_{\max } L^{3}}{\hat{Q}}\right)^{2}}}\right]^{\frac{1}{2}}
\end{aligned}
$$

Now let us try to estimate the value of the ratio $\left(C_{\max } L^{3}\right) / \hat{Q}$. Using the expressions (37) and (36) $)$ we have

$$
\frac{\hat{Q}}{L^{3}}=\frac{3}{\sqrt{2}}\left(\frac{\rho_{0}}{l_{s}}\right)^{3} e^{\theta / 2} \gg e^{-3 \theta / 2} e^{\theta / 2}=e^{-\theta}
$$

Also we have $C_{\max }=3^{4} /\left[2(4 \pi)^{3}\right]<1$. Now since we know that in order to have static bubbles we must have

$$
C_{\max }>\frac{\hat{Q}}{L^{3}} \gg e^{-\theta}
$$

The above condition can be rewritten as,

$$
1<\frac{C_{\mathrm{max}} L^{3}}{\hat{Q}} \ll C_{\max } e^{\theta}
$$


Since $\theta$ is a very large angle (50) gives us an estimate of the ratio $\left(C_{\max } L^{3}\right) / \hat{Q}$. Now when the ratio $\left(C_{\max } L^{3}\right) / \hat{Q}$ is greater than but close to 1 , then from (47) we find

$$
\cosh \tilde{\theta}_{s} \approx \cosh \tilde{\theta}_{\ell} \approx \sqrt{2}\left(\frac{C_{\max } L^{3}}{\hat{Q}}\right)
$$

and when $\left(C_{\max } L^{3}\right) / \hat{Q} \gg 1, \cosh \tilde{\theta}_{\ell}$ is still given by almost the same expression as given above in terms of $L^{3} / \hat{Q}$, but now $\cosh \tilde{\theta}_{s} \rightarrow 1$ and that implies $\tilde{\theta}_{s} \rightarrow 0$. So we have from (14),

$$
\tilde{\rho}_{0}=\frac{3}{4 \pi} \frac{L}{\cosh \tilde{\theta}_{\ell}} \sim \frac{\hat{Q}}{L^{2}}
$$

and therefore we have the size of the stable static bubble as,

$$
Z_{b} \sim \tilde{\rho}_{0} \cosh ^{\frac{1}{2}} \tilde{\theta}_{\ell} \approx\left(\frac{\hat{Q}}{L}\right)^{1 / 2}
$$

Now we find out the size of the dynamical bubble at the moment of its formation through closed string tachyon condensation as,

$$
Z_{\mathrm{dyn}}=\frac{\rho_{0}}{\sqrt{2}} e^{\theta / 2} \sim\left(l_{s} \frac{\hat{Q}}{L}\right)^{1 / 3}
$$

where in the last expression we have used (37) and (36). So, comparing (53) and (54) we get

$$
\frac{Z_{b}}{Z_{\mathrm{dyn}}}=\frac{\left(\frac{\hat{Q}}{L}\right)^{1 / 6}}{l_{s}^{1 / 3}} \gg 1
$$

In the last inequality we have first used (37) and (36) to obtain $\rho_{0} \approx \hat{Q}^{1 / 3}\left(l_{s} / L\right)^{4 / 3}$ and then from $\rho_{0} / l_{s} \gg e^{-\theta / 2} \approx l_{s} / L$ we have $\hat{Q} / L \gg l_{s}^{2}$. Note that eq.(155) tells us that the size of the dynamical bubble is much less than the stable static bubble and therefore, we expect that the dynamical bubble will evolve to eventually settle down to the stable static bubble. So, the black D4-brane will first decay to the dynamical bubble by closed string tachyon condensation and then evolve to settle down to the static stable bubble plus radiation and this would be the physical interpretation of the interpolation in this case.

Now let us next look at the cases $p=5,6$. For $p=5$ we have from (37), (36), (38) and (39) $\hat{Q} / L^{2}=\left(\rho_{0} / l_{s}\right)^{2} e^{\theta} \gg e^{-\theta} e^{\theta}=1$. On the other hand in order to have static bubble we need (see (26) $) \hat{Q} / L^{2}=\sinh \tilde{\theta} /\left(2 \pi^{2} \cosh \tilde{\theta}\right)<1$ and so, black D5-branes can decay only to dynamical bubbles by closed string tachyon condensation. Similarly for 
$p=6$, we have $\hat{Q} / L=\left(\rho_{0} / l_{s}\right) e^{3 \theta / 2} \gg e^{\theta}$. But, in order to have static bubble we need $\hat{Q} / L \sim \sinh \tilde{\theta}<e^{\tilde{\theta}} \sim e^{\theta}$ and therefore again we find that the bubble must be dynamical and expand to infinity.

In summary, for $p \leq 4$, when the black $p$-branes (which is compact with the compact directions having the sizes of the same order of magnitude) make transitions to the corresponding bubbles via closed string tachyon condensation, they can either decay to unstable static bubble and eventually settle down to the stable static bubble (this happens for $p<3 \sqrt{18}$ or they can directly decay to stable static bubble (this happens for $p=3$ ) or they can decay to dynamical bubble and then settle down to static stable bubble (this happens for $p=4)$. So, in all these cases of $p \leq 4$, the black branes can make transitions to static stable bubbles via closed string tachyon condensation and all the conditions for this to happen can be met as we have seen. Therefore, this would be the physical meaning of the interpolation of our solutions we constructed in section 2 in parameter space. Note that as we point out at the beginning of this section for $p=5,6$ cases, the interpolation cannot hold true even classically since the unstable static bubble will contract or expand under any small perturbation. The conclusion reached above via closed string condensation is entirely consistent with this fact and the bubbles formed this way must be dynamical and expand to infinity. In other words, the meaningful interpolation of our solutions exists only for $p \leq 4$ and for the branes with their brane directions compact and with all the compact sizes the same order of magnitude and this can be explained via the closed string condensation as described above.

Before closing this section, we remark that the black $p$-brane has a large entropy while the corresponding KK bubble at the moment of its formation, via closed string tachyon condensation, has no intrinsic entropy. Therefore, the closed string tachyon condensation must produce some radiation in addition to the bubble as pointed out in [4. This implies that the mass of the bubble must be less than the mass of the original black $p$-brane. We checked that this is indeed true.

\section{Origin of F-strings}

We have shown above that the supergravity solutions representing charged non-susy $p$ brane intersecting with chargeless non-susy 1-brane and 0-brane interpolate continuously between black D p-branes and KK BON when some parameters characterizing the solutions are varied from one set of values to another. The interpolation can be interpreted

\footnotetext{
${ }^{18}$ The unstable static bubble can also have the possibility to expand to infinity.
} 
physically as the transition from black D $p$-brane to KK BON only for $p \leq 4$, since only for these cases there exist static, locally stable bubbles as our initial data analysis suggests. The underlying mechanisms, as we have shown, for these transitions could be the closed string tachyon condensation for all $p \leq 4$.

As we have already noticed the interpolating solution (7) is well behaved at two end points, i.e. it is regular for the bubble solution and singular for the black $\mathrm{D} p$-brane solution at $\rho=0$ which is masked by a regular horizon. But for all other intermediate points the solutions have naked singularities at $\rho=\rho_{0}$. Two most natural questions which might arise at this point are

- How do we interpret the intermediate singular solutions?

- Where are the F-strings in our set up which when wind along the periodic coordinate $x^{1}$ give rise to tachyonic mode when the size of the circle reaches the string scale on the horizon?

To answer the first question we remark here that the intermediate solutions (7) (with the parameter values other than those given in (8), (9), (11), (12)) are all regular in the region $\rho_{0}<\rho \leq \infty$ and the naked singularity at $\rho=\rho_{0}$ reflects our inability to describe the system classically where the violent quantum process like the closed string tachyon condensation is occurring. It is very likely that quantum mechanically there are tachyon condensates [14] and no singularities, but classically we do not have a good description in general for the region $\rho \leq \rho_{0}$. To an observer far away from the core region (where the closed string tachyon condensation is occurring), only the long-range force would appear and so we have a classical description of the dynamics there and the description of the tachyon condensation can be viewed as an adiabatic process as discussed in the previous section. This long-distance description is just the family of the intermediate solutions with naked singularities. However, as we stressed, these singularities are just the artifact since their appearance is due to the extrapolation of our solutions valid only at long distance to the region where the description is invalid and where we actually have quantum process without any singularity. Here our remarks are very similar in spirit with the family of solutions discussed by Gross-Perry [2] and also is supported by the observations made in [20].

To answer the second question we point out that although our intersecting solutions (4) or (7) does not seem to contain F-strings to cause the closed string tachyon condensation, but we will show that (4) indeed contain chargeless non-susy F-strings in the sense defined in the Introduction and this is the reason we have the closed string tachyon condensation 
and consequently the transition from black-branes to KK BON 19 . Now in order to show this we will compare (44) with another known solutions constructed in ref.[15] representing charged non-susy F-strings intersecting with chargeless non-susy D p-branes. The solutions are given in eqs.(11) and (12) in [15] and have the forms,

$$
\begin{aligned}
d s^{2}= & \bar{F}^{\frac{1}{4}}(H \tilde{H})^{\frac{2}{7-p}}\left(\frac{H}{H}\right)^{-\left(2 \sum_{i=2}^{p} \gamma_{i}\right) /(7-p)}\left(d r^{2}+r^{2} d \Omega_{8-p}^{2}\right) \\
& +\bar{F}^{-\frac{3}{4}}\left(-d t^{2}+\left(d x^{1}\right)^{2}\right)+\bar{F}^{\frac{1}{4}} \sum_{i=2}^{p}\left(\frac{H}{\tilde{H}}\right)^{2 \gamma_{i}}\left(d x^{i}\right)^{2} \\
e^{2 \phi}= & \bar{F}^{-1}\left(\frac{H}{\tilde{H}}\right)^{2 \gamma_{1}}, \quad B^{(2)}=\frac{\sinh 2 \theta}{2}\left(\frac{\bar{C}}{\bar{F}}\right) d t \wedge d x^{1}
\end{aligned}
$$

with the parameter relation

$$
\frac{1}{2} \gamma_{1}^{2}+\frac{1}{2} \bar{\alpha}\left(\bar{\alpha}-\gamma_{1}\right)+\frac{2 \sum_{i>j=2}^{p} \gamma_{i} \gamma_{j}}{7-p}=\left(1-\sum_{i=2}^{p} \gamma_{i}^{2}\right) \frac{8-p}{7-p}
$$

Note that here we have labelled the various functions and the parameters differently to compare the possible differences with the configuration (44). Let us remark that here the $\mathrm{D} p$-branes are chargeless and also we don't have the presence of chargeless 0-brane but F-strings are charged. So in making identification, we need to send the F-string charge to zero which can be done by setting $\theta=0$. We also need to set $\gamma_{2}=\gamma_{3}=\cdots=\gamma_{p}=\gamma_{0}$ in order to make the directions $x^{2}, \ldots, x^{p}$ isotropic. This way we end up with solutions representing chargeless non-susy F-strings intersecting with chargeless non-susy $\mathrm{D} p$-branes as follows:

$$
\begin{aligned}
d s^{2}= & (H \tilde{H})^{\frac{2}{7-p}}\left(\frac{H}{H}\right)^{\frac{\bar{\alpha}}{4}-\frac{2(p-1) \gamma_{0}}{(7-p)}}\left(d r^{2}+r^{2} d \Omega_{8-p}^{2}\right) \\
& +\left(\frac{H}{H}\right)^{-\frac{3 \bar{\alpha}}{4}}\left(-d t^{2}+\left(d x^{1}\right)^{2}\right)+\left(\frac{H}{\tilde{H}}\right)^{\frac{\bar{\alpha}}{4}+2 \gamma_{0}} \sum_{i=2}^{p}\left(d x^{i}\right)^{2} \\
e^{2 \phi}= & \left(\frac{H}{\tilde{H}}\right)^{-\bar{\alpha}+2 \gamma_{1}},
\end{aligned}
$$

\footnotetext{
${ }^{19}$ There are many ways to see how such F-strings can appear. For example, the original non-isotropic solutions (1) contain chargeless $(p-1)$-brane, $(p-2)$-brane, upto 0 -brane if we take $q=0$ there. These chargeless branes are either brane-antibrane or non-BPS brane in the theory. The process of making the non-isotropic directions $x^{p}, x^{p-1}, \cdots$ upto $x^{2}$ isotropic is actually the process of elimination of the chargeless $(p-1)$-brane, $(p-2)$-brane upto 2-brane from the solution as mentioned earlier. This elimination can also be viewed as the end of annihilation process for these branes. The previous work [21, 22, 15] indicates that the annihilation of these branes will give rise to F-strings wrapping around $x^{1}$ non-perturbatively. In addition, the chargeless part of non-susy charged $\mathrm{D} p$ as well as part of the chargeless D-strings in this configuration can also give rise to F-strings when they annihilate.
} 
with the parameter relation

$$
\frac{1}{2} \gamma_{1}^{2}+\frac{1}{2} \bar{\alpha}\left(\bar{\alpha}-\gamma_{1}\right)+\frac{(p-1)(p-2) \gamma_{0}^{2}}{(7-p)}=\left(1-(p-1) \gamma_{0}^{2}\right) \frac{8-p}{7-p}
$$

Now to compare this with (4) we must put the $\mathrm{D} p$-brane charge to zero and also to remove the presence of 0 -branes we must put $p \delta_{1} / 8+2 \bar{\delta}=(p-8) \delta_{1} / 8+2 \bar{\delta}-2 \delta_{2} \Rightarrow \delta_{2}=-\delta_{1} / 2$. Then we have from (41)

$$
\begin{aligned}
d s^{2}= & (H \tilde{H})^{\frac{2}{7-p}}\left(\frac{H}{\tilde{H}}\right)^{\alpha \frac{p+1}{8}+\frac{p \delta_{1}}{8}+\frac{2(3-p) \bar{\delta}}{(7-p)}}\left(d r^{2}+r^{2} d \Omega_{8-p}^{2}\right) \\
& +\left(\frac{H}{\tilde{H}}\right)^{-\alpha \frac{7-p}{8}+\frac{p \delta_{1}}{8}+2 \bar{\delta}}\left(-d t^{2}+\left(d x^{1}\right)^{2}\right)+\left(\frac{H}{\tilde{H}}\right)^{-\alpha \frac{7-p}{8} \frac{(p-8) \delta_{1}}{8}+2 \bar{\delta}-2 \delta_{0}} \sum_{i=2}^{p}\left(d x^{i}\right)^{2} \\
e^{2 \phi}= & \left(\frac{H}{\tilde{H}}\right)^{\alpha \frac{3-p}{2}+\frac{\delta_{1}}{2}(4-p)-8 \bar{\delta}}
\end{aligned}
$$

and the parameter relation (the second equation of (3) ) takes the form:

$$
\frac{1}{2} \delta_{1}^{2}+\frac{1}{2} \alpha\left(\alpha+\frac{3}{2} \delta_{1}\right)+\frac{(p-1)\left[-\delta_{1}+(p-2) \delta_{0}\right] \delta_{0}}{7-p}=\left(1-\delta_{1}^{2} / 4-(p-1) \delta_{0}^{2}\right) \frac{8-p}{7-p}
$$

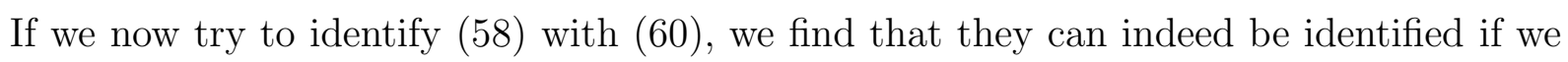
relate the parameters in the two solutions as follows:

$$
\begin{aligned}
\bar{\alpha} & =-\frac{2(p-1) \delta_{0}}{3}+\frac{(2-p) \delta_{1}}{6}+\frac{7-p}{6} \alpha \\
\gamma_{0} & =\frac{p-4}{3} \delta_{0}+\frac{p-8}{12} \delta_{1}-\frac{7-p}{12} \alpha \\
\gamma_{1} & =-\frac{4(p-1)}{3} \delta_{0}+\frac{5-p}{3} \delta_{1}+\frac{4-p}{3} \alpha .
\end{aligned}
$$

Using these relations, we can show that the parameter relation (59) indeed goes over to (61). This therefore shows that the supergravity configuration (41) or (17) contains a chargeless F-string causing the tachyon condensation as well as the transition to occur.

\section{Conclusion}

So, to summarize, in this paper we have explicitly constructed a class of type II supergravity solutions which interpolate between black D $p$-brane and KK BON when we vary the parameters characterizing the solutions from one set of values to another and obtained a physical meaning of this interpolation. The existence of such an interpolation 
in parameter space usually means a transition from black Dp-branes to KK BON. For this to be true indeed, the final bubble configuration must be static as well as stable at least classically. By performing a time symmetric initial data analysis we have shown that classically stable, static bubble can result only if $p \leq 4$ under certain conditions. For all other cases, we found that the bubbles are either static, but classically unstable or dynamical and therefore the interpolating solutions in these cases do not imply a real transition. After we understood this we looked for the underlying mechanism causing this transition. We found that except for $p=5,6$, in all other cases the interpolation can be understood as a real transition from black $p$-branes to the static KK BON caused by the closed string tachyon condensation. For $p<3$, the black branes first make transition to unstable static bubbles which eventually settle down to the stable static bubbles, for $p=3$, black D3-brane makes direct transition to the static stable bubble, whereas for

$p=4$, the black brane first makes transition to the dynamical bubble and then evolves finally to the static stable bubble. To have the transition to occur actually, we must have all the brane directions compact with their sizes of the same order of magnitude and the same must also be true for the corresponding bubbles. In all these cases the various conditions required for the closed string tachyon condensation to occur can be satisfied and therefore the interpolation we found in section 2 can be physically interpreted as the closed string tachyon condensation. Finally, in order to claim that our solutions in fact interpolate between black D $p$-brane (for $p \leq 4$ ) to the static $\mathrm{KK}$ BON or to dynamical bubbles by closed string tachyon condensation, we must show that the solutions contain F-strings which is not apparent in our intersecting solutions. We have explicitly shown this by comparing with another known solutions that our solutions indeed contain chargeless F-strings causing the closed string tachyon condensation which in turn leads the transition to occur.

\section{Acknowledgements:}

We wish to thank Hua Bai for collaboration at an early stage of this work, Sudipta Mukherji for useful discussions and the anonymous referee for the suggestions and comments which help us to improve the manuscript. JXL, ZLW and RJW acknowledge support by grants from the Chinese Academy of Sciences, a grant from 973 Program with grant No: 2007CB815401 and grants from the NSF of China with Grant No:10588503 and 10535060. 


\section{References}

[1] H. Bai, J. X. Lu and S. Roy, "Intersecting non-SUSY p-brane with chargeless 0-brane as black p-brane," JHEP 0701, 094 (2007) arXiv:hep-th/0610264.

[2] D. J. Gross and M. J. Perry, "Magnetic Monopoles In Kaluza-Klein Theories," Nucl. Phys. B 226, 29 (1983).

[3] E. Witten, "Instability Of The Kaluza-Klein Vacuum," Nucl. Phys. B 195, 481 (1982).

[4] G. T. Horowitz, "Tachyon condensation and black strings," JHEP 0508, 091 (2005) arXiv:hep-th/0506166.

[5] D. R. Green, E. Silverstein and D. Starr, "Attractor explosions and catalyzed vacuum decay," Phys. Rev. D 74, 024004 (2006) [arXiv:hep-th/0605047].

[6] S. F. Ross, "Winding tachyons in asymptotically supersymmetric black strings," JHEP 0510, 112 (2005) arXiv:hep-th/0509066.

[7] J. Scherk and J. H. Schwarz, "Spontaneous Breaking Of Supersymmetry Through Dimensional Reduction," Phys. Lett. B 82, 60 (1979).

[8] R. Rohm, "Spontaneous Supersymmetry Breaking In Supersymmetric String Theories," Nucl. Phys. B 237, 553 (1984).

[9] A. Adams, X. Liu, J. McGreevy, A. Saltman and E. Silverstein, "Things fall apart: Topology change from winding tachyons," JHEP 0510, 033 (2005) arXiv:hep-th/0502021.

[10] G. T. Horowitz and A. Strominger, "Black strings and P-branes," Nucl. Phys. B 360, 197 (1991).

[11] M. J. Duff and J. X. Lu, "Black and super p-branes in diverse dimensions," Nucl. Phys. B 416, 301 (1994) arXiv:hep-th/9306052.

[12] H. Bai, J. X. Lu and S. Roy, "Tachyon condensation on the intersecting braneantibrane system," JHEP 0508, 068 (2005) arXiv:hep-th/0506115.

[13] J. X. Lu and S. Roy, "Supergravity approach to tachyon condensation on the braneantibrane system," Phys. Lett. B 599, 313 (2004) arXiv:hep-th/0403147. 
[14] G. T. Horowitz and E. Silverstein, Phys. Rev. D 73, 064016 (2006) arXiv:hep-th/0601032.

[15] J. X. Lu and S. Roy, "Fundamental strings and NS5-branes from unstable D-branes in supergravity," Phys. Lett. B 637, 326 (2006) arXiv:hep-th/0508045.

[16] O. Sarbach and L. Lehner, "Critical bubbles and implications for critical black strings," Phys. Rev. D 71, 026002 (2005) arXiv:hep-th/0407265].

[17] R. Gregory and R. Laflamme, "Black strings and p-branes are unstable," Phys. Rev. Lett. 70, 2837 (1993) arXiv:hep-th/9301052]; "The Instability of charged black strings and p-branes," Nucl. Phys. B 428, 399 (1994) arXiv:hep-th/9404071].

[18] S. S. Gubser and I. Mitra, "Instability of charged black holes in anti-de Sitter space," arXiv:hep-th/0009126.

[19] R. G. Cai and Y. S. Myung, "Critical behavior for the dilaton black holes," Nucl. Phys. B 495, 339 (1997) arXiv:hep-th/9702159].

[20] M. Dine, A. Shomer and Z. Sun, "On Witten's instability and winding tachyons," JHEP 0612, 013 (2006) arXiv:hep-th/0607003.

[21] P. Yi, "Membranes from five-branes and fundamental strings from Dp branes," Nucl. Phys. B 550, 214 (1999) [arXiv:hep-th/9901159].

[22] O. Bergman, K. Hori and P. Yi, "Confinement on the brane," Nucl. Phys. B 580, 289 (2000) arXiv:hep-th/0002223. 